\title{
BMJ Open Evaluating the role of orange juice, HESPERidin in vascular HEALTH benefits (HESPER-HEALTH study): protocol for a randomised controlled trial
}

\author{
Marie-Anne Verny, ${ }^{1}$ Dragan Milenkovic, ${ }^{1}$ Nicolas Macian, ${ }^{2}$ Bruno Pereira, ${ }^{3}$ \\ Rémy Evrard, ${ }^{2}$ Caroline Gilcher, ${ }^{4}$ Christof B Steingass, ${ }^{4}$ Pascale Mosoni, ${ }^{5}$ \\ Cécile Gladine, ${ }^{1}$ Laurent-Emmanuel Monfoulet, ${ }^{1}$ Ralf Schweiggert, ${ }^{4}$ \\ Gisèle Pickering, ${ }^{2}$ Christine Morand (i) ${ }^{1}$
}

To cite: Verny M-A Milenkovic D, Macian N, et al. Evaluating the role of orange juice, HESPERidin in vascular HEALTH benefits (HESPER-HEALTH study): protocol for a randomised controlled trial. BMJ Open 2021;11:e053321. doi:10.1136/ bmjopen-2021-053321

- Prepublication history and additional supplemental material for this paper are available online. To view these files, please visit the journal online (http://dx.doi.org/10.1136/ bmjopen-2021-053321).

Received 10 May 2021 Accepted 25 0ctober 2021

Check for updates

(C) Author(s) (or their employer(s)) 2021. Re-use permitted under CC BY-NC. No commercial re-use. See rights and permissions. Published by BMJ.

For numbered affiliations see end of article.

Correspondence to Dr Christine Morand; christine.morand@inrae.fr

\section{ABSTRACT}

Introduction Although epidemiological studies associate the consumption of sugary beverages with adverse health effects, human experimental studies have demonstrated substantially different metabolic responses when $100 \%$ fruit juices are compared with artificial beverages. Fruit juices do not just provide sugars and associated calories, but they are also rich in bioactive compounds. Flavanones are bioactives specifically and abundantly found in citrus foods, with hesperidin as the major representative in sweet oranges. Flavanone intake has been associated with a lower incidence of mortality from cardiovascular disease (CVD). However, clinical evidence are too scarce to confirm the vasculoprotective effects of $100 \%$ orange juice (0J) presumably mediated by flavanones and thereby do not allow firm conclusions to be drawn about their efficacy. Methods and analysis The HESPER-HEALTH study aims to assess the efficacy of $0 \mathrm{~J}$ in improving vascular function and the contribution of hesperidin to these effects. This double-blind, randomised, controlled, crossover study will be carried out in 42 volunteers predisposed to CVD, based on age and on overweight. It includes three 6 week periods of consumption of $330 \mathrm{~mL} / \mathrm{d}$ of $0 \mathrm{~J}$ versus control drinks with and without hesperidin at a dose in agreement with a daily $0 \mathrm{~J}$ serving (approx. 200-215 mg). The primary outcome is endothelial function, assessed by flow mediated dilation, with measurements performed at fasting and postprandially in response to a challenge meal. The secondary outcomes include bioavailability and metabolism of flavanones, changes in other markers of vascular function, systemic biomarkers of cardiovascular risk, endothelial dysfunction and inflammation, vitamin $\mathrm{C}$ and carotenoids status, anthropometry and body composition, gut microbiota composition, nutrigenomic response and in oxylipin profiling.

Ethics and dissemination This ongoing study was approved by the Ethics committee Sud-Est III, Bron, France on 17 November 2020. The trial is registered on ClinicalTrials.gov. The results will be disseminated in peerreviewed journals.

Trial registration number NCT04731987; Pre-results.
Strengths and limitations of this study

- This randomised controlled dietary intervention is carried out to assess the effects of orange juice (0J) consumption on vascular function and to determine the contribution of hesperidin to these effects.

- This trial includes an analysis of hesperidin bioavailability and metabolism in biofluids to enable further correlation with the vascular response of individuals.

- The use of innovative approaches (ie, oxylipin profiling and nutrigenomics) will provide insights on the molecular mechanisms underlying the vascular effects.

- By including an analysis of the gut microbiota, this study will clarify interactions between $0 \mathrm{~J}$, hesperidin and the microbiome.

- The sensoriality of the two artificial control drinks differs from that of the fully natural $0 \mathrm{~J}$, which may constitute a study limitation.

\section{INTRODUCTION}

Recent epidemiological studies have associated the frequent consumption of sugarsweetened beverages with some adverse health effects, such as early death, weight gain and cancer ${ }^{12}$ Findings of such studies are often extrapolated to fruit juice consumption, including $100 \%$ fruit juices. However, these observational, epidemiological results contrast with experimental studies which have demonstrated clear differences in the metabolic response of the human body to fruit juices as compared with that seen for sugar-sweetened beverages. ${ }^{34}$

One characteristic of citrus foods is that they are a rich and exclusive source of dietary flavanones, a category of (poly)phenol compounds, mainly present as hesperidin 
in orange. Flavanone intake has been repeatedly associated with a lower incidence of mortality from cardiovascular disease (CVD).${ }^{56}$ Results from preclinical studies using different models of atherosclerosis also provide evidence for a role of citrus flavanones in cardiovascular protection, with a slowdown in atherosclerosis development. ${ }^{7}$ These atheroprotective effects have been related to the capacity of flavanones to modulate the expression of genes involved in cellular processes responsible for vascular dysfunction. ${ }^{8}$ Evidence of the vascular protective effects of citrus flavanones has been reported in few randomised controlled clinical trials. ${ }^{4-11}$ However, published trial with orange flavanones does not yet allow firm conclusions to be drawn about their efficacy to modulate vascular function, mainly due to the high degree of discrepancies between the study designs.

The ability of citrus flavanones to exert beneficial effects depends on their bioavailability. The gut microbiota plays a key role in flavanone absorption, because naturally occurring flavanones are present as molecules with glycosyl moieties, for example, a rutinosyl, that is, a $\alpha$-L-rhamnopyranosyl-( $1 \rightarrow 6)-\beta$-D-glucopyranosyl moiety in hesperidin. The sugar moiety of hesperidin must be hydrolysed by bacterial glycosidases to yield a format which the human body can absorb, that is, the aglycone, hesperetin. On release, hesperetin can be absorbed by intestinal cells or may be further catabolised into diverse phenolic compounds by microbial action in the colon. After absorption of hesperetin and its microbial catabolites, they enter the blood circulation and are subject to further human metabolism. For instance, hepatic metabolism includes several conjugation reactions, for example, sulphatation or glucuronidation. ${ }^{12}{ }^{13}$ Hence, the extent of hesperetin released in the colon, the formation of colonic catabolites and their subsequent absorption could largely depend on the gut microbial composition, which has been shown to vary between subjects. ${ }^{14}$ In agreement with this hypothesis, previous studies have reported a large interindividual variability in the urinary excretion level of flavanones ${ }^{1516}$ that could reflect differences in gut microbiota composition, but this remains to be demonstrated. A recent clinical study found that regular consumption of OJ can modulate the gut microbiota profile and these changes were associated with positive shifts in some metabolic outcomes. ${ }^{17}$

Dietary (poly) phenols and their circulating metabolites encompass a huge diversity of compounds ${ }^{18}$ whose health effects seem increasingly linked to their capacity to exert complex genomic modifications, such as changes to the expression of genes. ${ }^{19}$ In preclinical studies, flavanones have been shown to induce changes in the expression of a number of genes in aorta and endothelial cells that relate to inflammation and endothelial cell function, ${ }^{7}$ 20-22 revealing potential molecular mechanisms of action to explain their health properties. However, such mechanisms of action are still largely unexplored in humans. ${ }^{20}$ Based on a huge body of experimental results demonstrating the anti-inflammatory effects of polyphenols and related mechanisms, ${ }^{23}$ these compounds are suggested as key players in the protective effects of their food sources for chronic inflammatory diseases. However, in humans, consumption of polyphenol-rich foods induces subtle changes in inflammatory and oxidative status that can only typically be measured using sensitive techniques, such as the lipidomic profiling of oxylipins. ${ }^{24}$ Oxylipins are a superclass of lipid mediators comprising hundreds of metabolites which regulate a diversity of biological processes including inflammation, cell adhesion, migration and proliferation, blood clotting and vessel permeability. ${ }^{25}$

Based on this state of the art, the present human randomised, controlled, double-blind, crossover intervention conducted on subjects predisposed to CVD aims to establish a cause-and-effect relationship between hesperidin intake and the vascular protective effects of drinking OJ naturally rich in hesperidin. The study will also provide insights into the mechanisms responsible for the observed effects.

\section{STUDY OBJECTIVES \\ Primary objective}

The primary objective is to assess the effect of a subchronic consumption (6 weeks) of a naturally flavanone-rich OJ or a control drink supplemented with orange flavanones on endothelial function, by assessing flow-mediated dilation (FMD) in subjects with a predisposition to CVD, based on age, waist circumference and body mass index.

\section{Secondary objectives}

The secondary objectives are to:

- assess the effects of the intervention: (1) on other markers of vascular function using a range of wellestablished measurements in the macrocirculation (blood pressure, arterial stiffness) and in the microvasculature at skin level (microvascular reactivity by flow laser Doppler (FLD)); (2) on the postprandial endothelial response in response to a challenge meal; (3) on biomarkers of cardiovascular risk, endothelial dysfunction and inflammation; (4) on anthropometric parameters and body composition; (5) on the vitamin $\mathrm{C}$ and carotenoids status; (6) on the gut microbiota composition (microbial communities profiling),

- measure the bioavailability of hesperidin and its metabolites in biofluids (plasma, urine),

- ascertain the underpinning molecular mechanisms of the vascular responsiveness (nutrigenomic analysis, oxylipin profiling).

\section{METHOD AND ANALYSIS}

\section{Study design}

HESPER-HEALTH is a human dietary intervention study designed as a randomised, double-blind, controlled, crossover trial with three arms that will be conducted at the Clinical Investigation Center, Inserm 1405 (plateforme d'investigation clinique/centre d'investigation clinique 


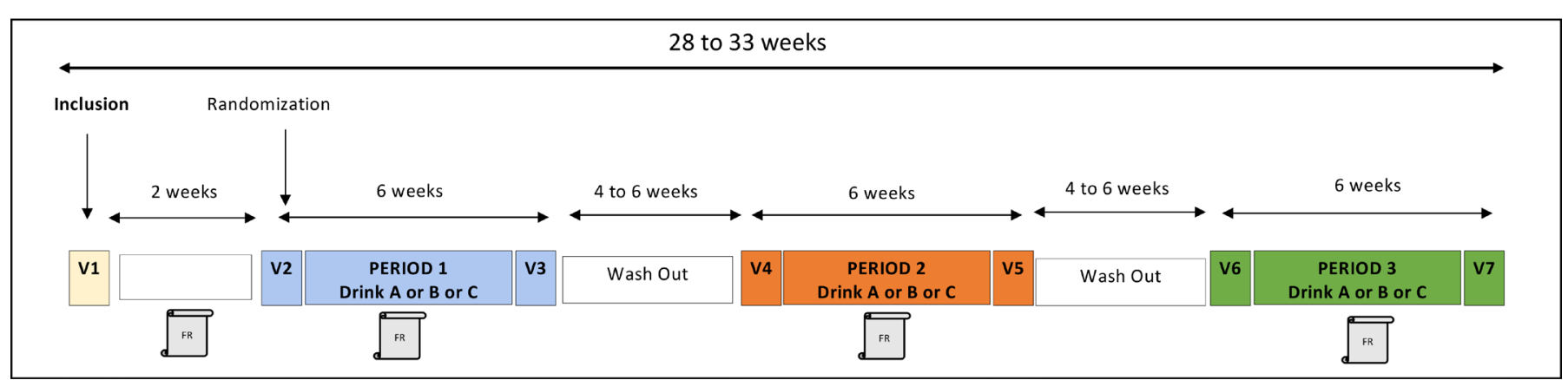

Figure 1 General scheme of HESPER-HEALTH study progress. V: visits in PIC/CIC; Drink A: OJ naturally rich in hesperidin; Drink B: control beverage with sugar concentrations identical to (A); Drink C: control beverage identical to (B) but supplemented with hesperidin at the level of (A); FR: 3-day food record. OJ, orange juice; PIC/CIC, plate-forme d'investigation clinique/centre d'investigation clinique.

(PIC/CIC)) of the University Hospital of ClermontFerrand, France. The sponsor is the University Hospital of Clermont-Ferrand. This trial will be carried out on subjects predisposed to CVD, based on age (40-65 years old) and on the overweight (waist circumference $\geq 80 \mathrm{~cm}$ for woman, $\geq 94 \mathrm{~cm}$ for man and with a BMI $\leq 30 \mathrm{~kg} / \mathrm{m}^{2}$ ). Forty-two participants will be recruited and will receive the three treatments in a random order: (A) a commercially available OJ naturally rich in hesperidin (ca. $600-650 \mathrm{mg} / \mathrm{L}$ ), (B) a control beverage with a total sugar concentration identical to $(\mathrm{A})$ and $(\mathrm{C})$ a control beverage identical to (B) but supplemented with hesperidin at the level present in the natural OJ (A).

For each volunteer, the study is divided in three identical experimental periods of 45 days. These periods include a 3-day run in period during which time specific dietary recommendations will be followed, samplings and measurements will be performed at home. This run in period will be followed by a 6 -week treatment period of consumption of one of the three beverages. A 4-6-week washout is planned between each of the three experimental periods. The protocol includes seven visits to $\mathrm{PIC} / \mathrm{CIC}$, including one visit (V1) at inclusion, and will last in total for 28-33 weeks (figure 1). Over the last 24 hours prior to each visit, volunteers will be asked to collect stool samples and 24 hours urine samples to assess gut microbiota composition and flavanone bioavailability and metabolism, respectively.

At the beginning and end of each experimental period, overnight-fasted volunteers will be invited to attend the PIC/CIC (visits V2-V7) for vascular function tests, blood sampling, anthropometric measurements and body composition analysis. Blood will be sampled for further assessment of plasma flavanones including metabolites, carotenoids and vitamin C, oxylipin profiling, systemic biomarkers of endothelial activation and inflammation, metabolic parameters and for the analysis of the nutrigenomic response.

At each visit (V2-V7), any adverse events (AE) will be followed up. In case of occurrence of a serious AE, the sponsor will be notified and in return he will notify the competent authorities. All unexpected serious AE are reported in an annual safety report. After measurements and blood collection in the fasting state, a challenge meal together with the respective study drink will be administered to all subjects before further exploration. This will enable evaluation of the acute postprandial effects (at $\mathrm{T}+3$ hour, $\mathrm{T}+6$ hour) of the study products on endothelial function (V2, V4, V6), to also be repeated after the 6 weeks intervention period (V3, V5, V7). The challenge meal will consist of fresh cream, sucrose and milk proteins, providing $900 \mathrm{kcal}-\mathrm{a}$ pro-oxidant and proinflammatory meal that is known to induce a transient endothelial dysfunction during the post prandial period. ${ }^{26}$

During each experimental period, volunteers will be asked not to eat citrus foods and to consume no more than $250 \mathrm{~mL} /$ day of polyphenol-rich beverages (coffee, tea, fruit juices, wine, cocoa). To check that volunteers do not change their eating habits during the study, for each experimental period, they will be asked to complete food records over three consecutive days defined by the investigator. The first food record will be completed prior to visit V2 to PIC/CIC and the two others at mid-term of each intervention period. On the 2 days preceding each visit at the PIC/CIC, volunteers will be asked not to consume polyphenol-rich foods and beverages. The day before each visit (V2-V7) at the PIC/CIC, they will have to consume exactly the same dinner without polyphenols at 20:00 hours. To ensure a good understanding of all these dietary instructions, participants meet a dietician during the inclusion visit (V1).

\section{Inclusion and exclusion criteria}

These criteria are listed in the table 1.

The reasons for a premature cessation of the study include withdrawal of consent, significant deviation from the protocol, incidental illness, occurrence of a serious adverse event, intolerance to the tested products, antibiotic treatment during the study, a non-observance of the nutritional protocol declared by the subject or highlighted by food records. 
Table 1 Inclusion and exclusion criteria for participation in HESPER-HEALTH study

\begin{tabular}{ll}
\hline Inclusion criteria & Exclusion criteria \\
\hline Man or woman & Treated prediabetic or diabetic \\
40-65 years (inclusive) & Treated for hypertension \\
Postmenopausal woman & Use of statins or other medications for lowering cholesterol \\
$\begin{array}{l}\text { Overweight (waist circumference } \geq 80 \mathrm{~cm} \text { for } \\
\text { women and } \geq 94 \mathrm{~cm} \text { for men, with } \mathrm{BMI} \leq 30)\end{array}$ & $\begin{array}{l}\text { Treated with antibiotics, antifungals, probiotics or prebiotics in the } 3 \text { months } \\
\text { before the enrolment }\end{array}$ \\
& Menopausal hormone therapy
\end{tabular}

Ability to give informed consent to participate Diagnosed gastrointestinal illness

in research.

Willingness to accept randomisation and undergo the testing and intervention

Any serious medical condition that precludes safe participation in the study procedures and deliver stool, blood and urine samples for testing

No aversion or intolerance to citrus foods

Accept to limit their total intake of flavonoid rich beverages (tea, coffee, cocoa, wine, fruit juice) to $250 \mathrm{~mL} /$ day

\section{History of eating disorders}

Digestive disorders with diarrhoea during the 3 months preceding the beginning of the study

Self-declared vegetarian, vegetarian, vegan

History of substance or alcohol abuse

Involvement in a weight loss programme within the three past months or who had a bariatric surgery

Current smokers (within the last 30 days)

Use of dietary supplements currently) or in the past 1 month

Declarative strenuous exercise greater than 6 hours per week

BMI, body mass index.

\section{Study products}

Three study beverages will be used in crossover and double-blinding conditions. These include (A) an OJ from a commercially available OJ concentrate (Sucocítrico Cutrale, Araraquara, São Paulo, Brazil) containing approx. 600-650 mg hesperidin per L, (B) a control drink with a total sugar concentration identical to that of $(\mathrm{A})$ and $(\mathrm{C})$ a control drink identical to (B) but supplemented with hesperidin at the level found in the OJ (figure 2). The daily administered dose will be $330 \mathrm{~mL}$ to be distributed over two intakes $(2 \times 165 \mathrm{~mL})$-one in the morning during breakfast and the second during lunch. This will correspond to ca. 200-215 mg hesperidin per day (drinks A and $\mathrm{C}$, respectively).

The control beverages (B) and (C) will be made isocaloric to (A), all containing an identical total sugar concentration (ca. $9.0 \mathrm{~g} / 100 \mathrm{~mL}$ ), comprising glucose, fructose and sucrose. Thus, the amount of total sugar provided daily by each drink will amount to ca. $30 \mathrm{~g}$. All drinks will contain citric acid at similar levels. To match the visual appearance of the OJ, food colourants as well as a clouding agent will be added to the control drinks. Flavour will be matched by adding a natural orange aroma to all beverages $(\mathrm{A}-\mathrm{C})$, including the $\mathrm{OJ}$ from concentrate to which orange aroma addition is required by European law. All the beverages will be pasteurised according to commercial practice and filled into $330 \mathrm{~mL}$ brown glass bottles with no visual distinction possible between bottles of different drinks. Despite careful colour and flavour matching, both the hesperidin-free (B, placebo) and the hesperidin-rich $(\mathrm{C})$ control drink may be differentiated sensorially from the OJ by attentive subjects. In contrast, subjects and clinic staff will be unable to identify which of the artificial control drinks (B) and (C) is the one rich in or free from hesperidin.

Finally, bottles will be labelled and packaged for each crossover period in a blinded fashion on the basis of the randomisation schedule. They will be stored at $+4^{\circ} \mathrm{C}$ until distribution to the participants. Beverages will be distributed to volunteers at the first visit of each experimental period (V2, V4, V6) in bags containing 48 bottles per period. Volunteers will be asked to store the bottles in a dark place at room temperature or cooler. The produced beverages will be analytically characterised in detail, including, for example, the levels of potassium, ${ }^{27}$ carotenoids $^{28}$ and soluble, insoluble ${ }^{14}$ and total hesperidin. ${ }^{29}$ Hesperidin levels in drinks (A) and (C) as well as levels of ascorbic and dehydroascorbic acid (vitamin $\mathrm{C}$ ) in the OJ (A) will be monitored over the entire study period. Colony forming units of yeasts, total viable counts, lactic acid bacteria and moulds will also be assessed to ensure the microbiological safety of the products. To assess compliance, participants will be asked to report daily on diaries their consumption of the study beverages and 


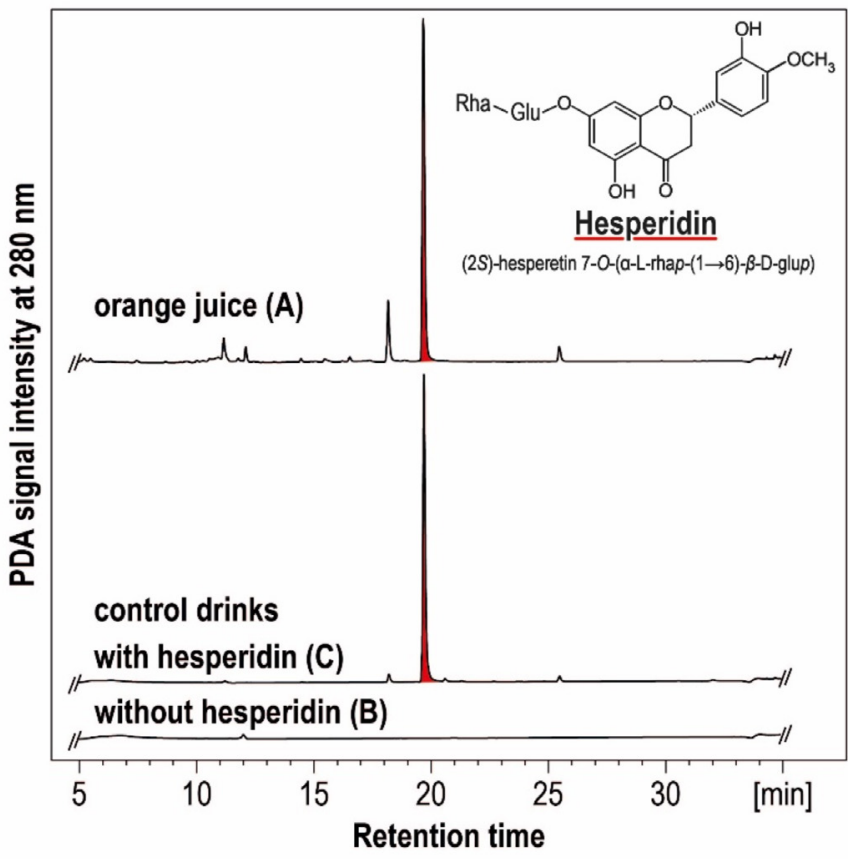

Figure 2 HPLC-DAD chromatogram of an OJ from a commercially available $\mathrm{OJ}$ concentrate naturally rich in hesperidin (A), a control beverage with an identical total sugar concentration (B) and a control beverage additionally supplemented with hesperidin $(C)$. Flavonoids were extracted according to IFU ${ }^{29}$ and analysed by HPLC-DAD using a C18 column $(250 \times 4.6 \mathrm{~mm}$, particle size $5.0 \mu \mathrm{m}$, Kinetex, Phenomenex, Aschaffenburg, Germany) and an acetonitrilebased elution gradient. HPLC-DAD, high-pressure liquid chromatography - diode array detection; OJ, orange juice.

to return the dairies and the empty and non-consumed bottles at the end of each period (V3, V5, V7).

\section{Assignment of intervention}

Randomisation and allocation

Subjects will be randomly assigned to treatment groups according to a pre-established list of randomisation designed by a clinical research supervisor, independently from the investigators and the sponsor. The treatment number will be allocated by order of entrance in inclusion. Subjects will be randomised to receive drink A, B or C using a Latin-square random design. Subject allocation to treatments will take place after inclusion and will be based on a computer-generated randomisation list. Access to the randomisation list will be restricted to staff performing this task. The randomisation number will be allocated by order of entrance in inclusion. Under normal circumstances, the blinding should not be broken until all subjects have completed the trial and the database will be locked. However, the blinding can be broken should a specific emergency treatment at the site require knowledge of the treatment status of the subject. In such a case, the investigator can, using a sealed envelop and following an internal procedure, reveal the treatment assignment through the randomisation plan and then inform the sponsor.

\section{Participant eligibility}

Forty-two volunteers will be recruited from the existing PIC/CIC volunteer's database, by announcement in local press and media by paper and digital poster campaigns and by social networks. Each subject who meets the recruitment criteria (table 1 ) will be preselected by the investigator and will be given a detailed explanation of the protocol. Participant who agree to participate will have a first visit (V1, figure 1) at the PIC/CIC to provide their written consent to participate and to have a medical examination, an interview with a dietitian and a blood check-up to ensure that all clinical criteria are met.

\section{Sample size}

Sample size calculation has been performed based on both our experience in FMD measurement and on the FMD response (main criteria) observed in previous dietary interventions. ${ }^{30}{ }^{31}$ The targeted statistical power was based on interindividual variability for FMD measurement of the operator $(\mathrm{SD}=1.9 \%)$. Aiming for a statistical power greater than $80 \%$ and a two-tailed type I error at 0.017 (to take into account the three comparisons to be considered), the total number of subjects required to provide sufficient power to detect a minimal absolute difference of FMD equals $1.6 \%$ in a three-sequences crossover study (with an intraclass correlation coefficient at 0.5 ) is 36 . Assuming unforeseen dropouts and follow-up losses, 42 participants will be recruited.

\section{Study assessments}

The time table of the measurements that will be performed in the HESPER-HEALTH study is presented in figure 3. The outcome assessors and data analysts will be blinded for treatments.

\section{Primary outcome}

Endothelial function is assessed using the non-invasive technique FMD which constitutes the gold standard to evaluate vascular endothelial function in humans. The FMD technique measures the diameter of the brachial artery by ultrasound before and after increasing shear stress by inducing a reactive hyperaemia, with the degree of dilation reflecting mostly the arterial endothelial nitric oxide release. The procedure for the FMD measurement is fully compliant with the reference method described by Corretti et al..$^{32}$ FMD is measured on the left brachial artery above the antecubital fossa using a high-resolution ultrasound system with a 7-12 MHz linear array 190 transducer (Vivid S5, GE Healthcare, Versailles, France). The use of a mechanical arm device (Vascular Imaging, Amsterdam, Netherlands) allows precise movements of the probe in the three dimensions. Images are analysed using automated edge detection software 197 (Hemodyn 3M apparatus, Dinap SRL, Buenos Aires, Argentina). FMD will be assessed at V2, V3, V4, V5, V6 and V7 under both fasting and postprandial conditions. 


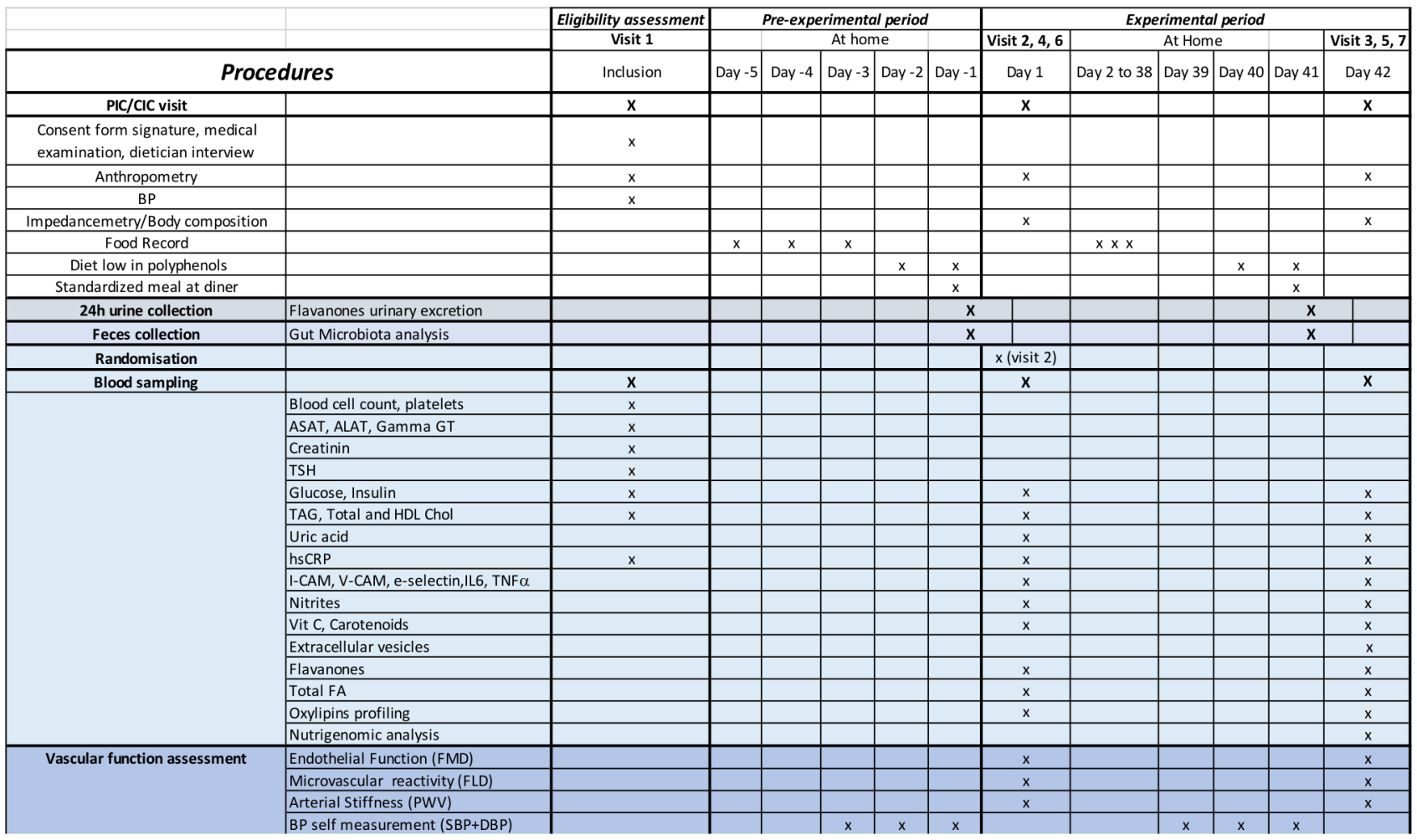

Figure 3 Time table of the conduct and measurements of the HESPER-HEALTH study. The crosses indicate the visits and types of samples taken $(X)$ and the recommendations and measurements made for each of them $(x)$. ALAT, alanine amino transferase; ASAT, aspartate amino transferase; BP, blood pressure; DBP, diastolic blood pressure; FLD, flowmetry by laser Doppler; FMD, flow mediated dilatation; Gamma GT, gamma glutamyl transferase; HDL-chol, high-density lipoprotein cholesterol; hsCRP, high sensitivity C reactive protein; ICAM, intercellular adhesion molecule; IL-6, interleukin-6; PIC/CIC, plateforme d'investigation clinique/centre d'investigation clinique; PWV, pulse wave velocity; SBP, systolic blood pressure; TAG, triacylglycerol; TNF $\alpha$, tumour necrosis factor alpha; TSH, thyroid stimulating hormone; VCAM, vascular cell adhesion molecule.

\section{Secondary outcomes}

\section{Other vascular function measurements}

Endothelial function in the microcirculation will be assessed using FLD, which is a non-invasive and validated technique for continuous measurement of the endothelial dependent microvascular reactivity. The laser-Doppler system PeriFlux 5010 (Perimed) is used at the level of the hand to follow the response to a reactive hyperaemia induced by a temporary occlusion of the brachial artery, using the same stimulus as for FMD measurement. FLD will be assessed during V2, V3, V4, V5, V6 and V7 in fasting and postprandial conditions.

Arterial stiffness will be evaluated by the carotidfemoral pulse wave velocity (PWV) which is calculated from measurements of pulse transit time and the distance travelled between the carotid and femoral arteries using a validated non-invasive device (SphygmoCor; AtCor Medical). PWV will be assessed after fasting at V2, V3, V4, $\mathrm{V} 5, \mathrm{~V} 6$ and V7.

Blood pressure will be monitored by subjects at home using tensiometers (Microlife BP A200, Microlife) which will be loaned to them for the study duration. They will be asked to perform three repeated measurements in fasting condition during the three consecutive days preceding their visits to the PIC/CIC (V2, V3, V4, V5, V6 and V7) and to record obtained values on a dedicated data sheet.

\section{Flavanone metabolism and bioavailability in biofluids}

Phase 2 metabolites of the Citrus flavanones and their microbial-derived catabolites of flavanones including conjugated forms like glucuronidated and sulfated ones will be analysed in plasma and 24hours urine samples according to the method based on those reported by Aschoff $e t a l^{5}$ and Mullen et $\mathrm{al} .{ }^{33}$ For plasma and urine analyses, acetonitrile and methanol will be used as extraction solvents as described in detail by Mullen et $a l^{33}$ A subset of urine and plasma samples will be analysed after enzymatic hydrolysis with glucuronidase and sulfatase as described by Aschoff et al. ${ }^{15}$ Identification and quantitation will be done by UHPLC-DAD-ESI-MS/MS (ultra-high-performance liquid chromatography diode array detector electrospray ionisation tandem mass spectrometry) for all samples collected (urine) or sampled (plasma) at V2, V3, V4, V5, V6 and V7.

\section{Systemic biomarkers related to CVD risk, endothelial dysfunction and inflammation}

These include metabolic parameters (plasma glucose, insulin, triacylglycerol, total cholesterol, high-density lipoprotein cholesterol, uric acid that will be measured by spectrometric and enzymatic methods; total fatty acids, by gas chromatography), soluble adhesion molecules and inflammatory markers (intercellular adhesion molecule, vascular cell adhesion molecule and e-selectin, interleukin-6, tumour 
necrosis factor alpha, high sensitivity-C reactive protein, by using Elisa assays), plasma nitrites (by chemiluminescence) and the release of endothelial extracellular vesicles (by flux cytometry). These analyses will be performed in blood sampled at V2, V3, V4, V5, V6 and V7.

\section{Carotenoids and vitamin C status}

Carotenoids will be quantitated from human plasma after a protein-crash with ethanol and repeated extraction with hexane using the method described by Aschoff $e t$ $a l^{28}$ Vitamin C status will be quantified in deproteinised plasma by HPLC-fluorescence detection as previously described. ${ }^{34}$ These measures will be performed in blood sampled at V2, V3, V4, V5, V6 and V7.

\section{Anthropometry and body composition assessment}

BMI, waist to height ratio (waist circumference/height) and the percentages of fat mass, lean mass and water (obtained using a multifrequency Bioelectrical Impedance Analyser) will be determined. These parameters will be recorded at V2, V3, V4, V5, V6 and V7.

\section{Gut microbiota composition assessment}

The composition of the gut microbiota will be determined by $16 \mathrm{~S}$ metabarcoding. This method involves the amplification by PCR of variable regions of the $16 \mathrm{~S}$ rDNA gene from faecal DNA followed by the preparation of DNA libraries and high throughput next-generation sequencing (Illumina technology). Analysis will be completed by an absolute quantification by qPCR of target groups of the human gut microbiota considered as beneficial to the host or involved in polyphenol metabolism. These analyses will be performed in faeces samples collected during the 12 hours preceding V2, V3, V4, V5, V6 and V7.

\section{Mechanistic outcomes}

In the HESPER-HEALTH study, we aim to explore the molecular mechanisms of action underlying vascular effects of OJ and hesperidin consumption. To this end, we propose to perform global and integrated analyses of expression of genes (nutrigenomic analysis) in the blood and to analyse the oxylipin profiling to identify changes in biological processes involved in inflammation and vascular dysfunction.

Nutrigenomic response assessment: RNA will be extracted from whole blood using PAXgene Blood RNA System. Global gene expression profile of both protein coding and protein non-coding RNAs (miRNAs, snoRNAs, lncRNAs) will be assessed using a microarray approach. For genes identified as differentially expressed, integrated multiomic bioinformatic analyses will be performed to identify gene ontologies, gene network, cellular pathways and interactions between different types of RNAs. Potential transcription factors involved will be searched and capacity of flavanone metabolites to bind to transcription factors as well as cell signalling proteins regulating their activity will be predicted using 3D docking analysis.
Analysis will be performed from blood sampled at V3, V5 and V7.

Oxylipin profiling: a comprehensive assessment of circulating total oxylipins (free and esterified forms) will be performed using a targeted and quantitative MS-based method (LC-MS/MS) as described previously. ${ }^{35}$ Briefly, EDTA plasma will be mixed with 22 internal standards and an antioxidant solution preventing artificial oxylipin production during sample processing. Then, total oxylipins will be extracted using solid phase extraction following protein precipitation and alkaline hydrolysis. Extracted oxylipins will then be measured using electrospray ionisation in negative ion mode and multiple reaction monitoring. Analysis will be performed in plasma sampled at V2, V3, V4, V5, V6 and V7.

\section{Statistical analyses}

All analyses will be performed with Stata software (V.15, Stata, College Station, Texas, USA) before the breaking of the randomisation code, according to International Conference on Harmonization-Good Clinical Practice guideline. Continuous variables will be presented, according to their statistical distribution, as mean and SD, or median and IQR. The Shapiro-Wilk test will be used to assess normality. Categorical data will be presented as exact number and percentage.

The primary outcome will be analysed using randomeffects model (ie, mixed linear model for continuous dependent variable) for a three-treatment crossover study. The statistical model includes treatment group, sequence, period, carryover on FMD baseline value as fixed effects. Subject nested in sequence will be included in the model as a random effect. A Sidak's type I error will be applied to take into account multiple comparisons. The treatment group $\times$ period interaction will be studied. If this interaction is not significant, the main effect of treatment will be assessed. If this interaction is significant, there will be a particular focus on the first period. The normality of residuals from random-effects model will be studied as aforementioned, with the Shapiro-Wilk statistic and visual inspection of residual plots. If appropriate, a transformation (eg, logarithmic) of the primary outcome could be proposed to achieve its normality. The results will be expressed with effect-sizes and $95 \%$ CI.

The primary analysis will be completed by multivariable approach using the statistical model described above with, additionally, covariates determined according to univariate results and clinical relevance, including primarily waist circumference, body mass index (BMI), weight at enrolment, age, gender and energy intake. The other continuous outcomes will be analysed with the same statistical analysis plan. For categorical data, generalised mixed linear modelling will be carried out. Any change in the current statistical analysis plan during the study will be noted in the study records. 


\section{ETHICS AND DISSEMINATION}

\section{Approval}

In accordance with the Declaration of Helsinki and French regulations on clinical trials, the study was evaluated by an independent ethics committee, chosen at random by the French ministry of research, namely the 'Comité de Protection des Personnes Sud-Est III, Bron, France' (registration number: 20.10.15.60521). The approval of the ethics committee was obtained on 17 November 2020. The first inclusion was on 24 February 2021. The study is declared to the French National Agency for the Safety of Medicines (ID-RCB : 2020-A01985-34). Any substantial change in the protocol or in the informed consent form will be presented to both authorities. The trial is also registered on ClinicalTrials.gov (NCT04731987).

\section{Consent}

Subjects will be informed by a fair and accessible form approved by the ethics committee. Subjects will be free to ask any question on all aspects of the study before giving the consent and informed that they are free to withdraw from the study at any time. The investigator will ensure that the written consent obtained from subjects prior to their participation in the study is free and informed (online supplemental data 1) .

\section{Data quality and management}

The data collected will be anonymised by a subject code assigned to each participant. All data from the interview, clinical examination and explorations will be reported in a paper Case Report Form (CRF) by experimented and trained study managers of the PIC/CIC and will constitute the data trial kept confidential. The database of the study will be designed and managed using the Research Electronic Data Capture (REDCap, Vanderbilt University), a dedicated software allowing a secure and local storage of the data and also an audit trail by user authentication. Data entries will be made from the paper CRF with a double entry procedure. After reconciliation of the two entries and elimination of transcription errors, data validation will be carried out to resolving discrepancies by the data manager. Then, database will be locked and securely sent to the biostatistician.

According to the 'minimal risks and constraints' ranking of this study by the ethics committee, the monocentric design and the low risk calculated by the sponsor, there are no data monitoring committee and no interim analyses planned for this study. Audits or inspections concerning the trial or investigation team activities could be carried out at any time.

Data availability statement: data not available due to legal restriction.

\section{Dissemination}

The results will be communicated to participants, in peerreview journals, and presented at international conferences in the domain of Food, Nutrition \& Health.
Patient and Public Involvement statement: patients and the public were not involved in the design or conduct of this protocol.

\section{DISCUSSION}

The controlled randomised HESPER-HEALTH study will use reliable and sensitive clinical biomarkers of human health to provide a comprehensive picture of the effects of a flavanone-rich $\mathrm{OJ}$ on different components of vascular function. It will also produce mechanistic insights to support the contribution of citrus flavanones in vascular health. Together, the results of the study will strengthen the level of evidence of the link between flavanone intake and the effects of a moderate consumption of $\mathrm{OJ}$ on vascular endpoints in humans. The results may also improve understanding of the role of gut microbiota in the interindividual variability in the absorption and metabolism of citrus flavanones, and the putative prebiotic-like effect of flavanone-rich foods. The results from HESPERHEALTH could help health professionals communicate science-driven dietary advice about fruit juice consumption to their patients. They should also be useful for the citrus sector to encourage the selection of orange varieties naturally rich in hesperidin and to provide healthfocused guidance on how to adapt processing methods to produce fruit juice richer in readily available flavanones.

\section{Author affiliations}

${ }^{1}$ Clermont Auvergne, INRAE, UNH, Clermont-Ferrand, France

${ }^{2}$ Platform of Clinical Investigation Department, University Hospital Clermont-Ferrand, INSERM CIC 1405, Clermont-Ferrand, France

${ }^{3}$ Clinical Research and Innovation Department, Biostatistics unit, University Hospital Clermont-Ferrand, Clermont-Ferrand, France

${ }^{4}$ Chair of Analysis \& Technology of Plant-based Foods, Department of Beverage

Research, Geisenheim University, Geisenheim, Germany

${ }^{5}$ Clermont Auvergne, INRAE, MEDIS, Clermont-Ferrand, France

Acknowledgements We thank the medical team of the PIC/CIC for carrying the clinical investigations and Lise Bernard at the Central Pharmacy of the University Hospital Clermont-Ferrand. We thank Dr Volker Herdegen, Dietmar Gürster, Caroline Grimm and Tobias Schardt from Eckes Granini (Niederolm, Germany) for providing a pilot production line and helping to produce and package the drinks to be tested. We thank Peter Bach, Tim Dreifke, Paul Luka Dreis and Anna-Maria Schmelzer (all Geisenheim University) for their technical assistance during beverage development and production. We thank Sucocítrico Cutrale (Araraquara, São Paulo, Brazil) for donating the $0 \mathrm{~J}$ concentrate. Döhler (Darmstadt, Germany) is gratefully acknowledged for donating the colorants, clouding agent and flavouring used in control drink development and production. HealthTech Bio Actives (Beniel, Spain) is acknowledged for providing the hesperidin formulation.

Contributors M-AV, DM, NM, RE, CGi, CBS, PM, CGI, L-EM, RS, GP and CM contributed to the conceptualisation, design and implementation of this research protocol. BP led to the development of the statistical analysis plan. All authors read and approved the final manuscript.

Funding This work was supported by a consortium of orange producers, juice manufacturers and packaging companies based in Europe and Brazil under the umbrella of the European Fruit Juice Association (AIJN). A representative from AIJN will give his opinion before any communication or publication.

Disclaimer The views presented in this paper are those of the authors. Funding companies have played no role in the study beyond providing financial support.

Competing interests None declared.

Patient consent for publication Not applicable. 
Provenance and peer review Not commissioned; externally peer reviewed.

Supplemental material This content has been supplied by the author(s). It has not been vetted by BMJ Publishing Group Limited (BMJ) and may not have been peer-reviewed. Any opinions or recommendations discussed are solely those of the author(s) and are not endorsed by BMJ. BMJ disclaims all liability and responsibility arising from any reliance placed on the content. Where the content includes any translated material, BMJ does not warrant the accuracy and reliability of the translations (including but not limited to local regulations, clinical guidelines, terminology, drug names and drug dosages), and is not responsible for any error and/or omissions arising from translation and adaptation or otherwise.

Open access This is an open access article distributed in accordance with the Creative Commons Attribution Non Commercial (CC BY-NC 4.0) license, which permits others to distribute, remix, adapt, build upon this work non-commercially, and license their derivative works on different terms, provided the original work is properly cited, appropriate credit is given, any changes made indicated, and the use is non-commercial. See: http://creativecommons.org/licenses/by-nc/4.0/.

\section{ORCID iD}

Christine Morand http://orcid.org/0000-0001-8128-1032

\section{REFERENCES}

1 Chazelas E, Srour B, Desmetz E, et al. Sugary drink consumption and risk of cancer: results from nutrinet-sante prospective cohort. BMJ 2019;366:12408.

2 Collin LJ, Judd S, Safford M, et al. Association of sugary beverage consumption with mortality risk in US adults: a secondary analysis of data from the REGARDS study. JAMA Netw Open 2019;2:e193121.

3 Büsing F, Hägele FA, Nas A, et al. High intake of orange juice and cola differently affects metabolic risk in healthy subjects. Clin Nutr 2019;38:812-9.

4 Morand C, Dubray C, Milenkovic D, et al. Hesperidin contributes to the vascular protective effects of orange juice: a randomized crossover study in healthy volunteers. Am J Clin Nutr 2011;93:73-80.

5 Cassidy A, Bertoia M, Chiuve S, et al. Habitual intake of anthocyanins and flavanones and risk of cardiovascular disease in men. Am J Clin Nutr 2016;104:587-94.

6 Mizrahi A, Knekt P, Montonen J, et al. Plant foods and the risk of cerebrovascular diseases: a potential protection of fruit consumption. Br J Nutr 2009;102:1075-83.

7 Chanet A, Milenkovic D, Claude S, et al. Flavanone metabolites decrease monocyte adhesion to TNF- $\alpha$-activated endothelial cells by modulating expression of atherosclerosis-related genes. Br J Nutr 2013;110:587-98.

8 Morand C, Barber-Chamoux N, Monfoulet L. Dietary (poly)phenols and vascular health. In: Recent advances in polyphenol research. vol. 6, 2019.

9 Habauzit V, Verny M-A, Milenkovic D, et al. Flavanones protect from arterial stiffness in postmenopausal women consuming grapefruit juice for 6 Mo: a randomized, controlled, crossover trial. Am J Clin Nutr 2015;102:66-74.

10 Li L, Lyall GK, Martinez-Blazquez JA, et al. Blood orange juice consumption increases flow-mediated dilation in adults with overweight and obesity: a randomized controlled trial. J Nutr 2020;150:1-8.

11 Valls RM, Pedret A, Calderón-Pérez L, et al. Effects of hesperidin in orange juice on blood and pulse pressures in mildly hypertensive individuals: a randomized controlled trial (Citrus study). Eur J Nutr 2021;60:1277-88.

12 Cani PD, Everard A. Talking microbes: when gut bacteria interact with diet and host organs. Mol Nutr Food Res 2016;60:58-66.

13 Tomás-Barberán FA, Selma MV, Espín JC. Interactions of gut microbiota with dietary polyphenols and consequences to human health. Curr Opin Clin Nutr Metab Care 2016;19:471-6.

14 Vallejo F, Larrosa M, Escudero E, et al. Concentration and solubility of flavanones in orange beverages affect their bioavailability in humans. $J$ Agric Food Chem 2010;58:6516-24.
15 Aschoff JK, Riedl KM, Cooperstone JL, et al. Urinary excretion of citrus flavanones and their major catabolites after consumption of fresh oranges and pasteurized orange juice: a randomized crossover study. Mol Nutr Food Res 2016;60:2602-10.

16 Tomás-Navarro M, Vallejo F, Sentandreu E, et al. Volunteer stratification is more relevant than technological treatment in orange juice flavanone bioavailability. J Agric Food Chem 2014;62:24-7.

17 Fidélix M, Milenkovic D, Sivieri K, et al. Microbiota modulation and effects on metabolic biomarkers by orange juice: a controlled clinical trial. Food Funct 2020;11:1599-610.

18 Kay CD, Clifford MN, Mena P, et al. Recommendations for standardizing nomenclature for dietary (poly)phenol catabolites. Am J Clin Nutr 2020;112:1051-68.

19 Krga I, Milenkovic D, Morand C, et al. An update on the role of nutrigenomic modulations in mediating the cardiovascular protective effect of fruit polyphenols. Food Funct 2016;7:3656-76.

20 Milenkovic D, Deval C, Dubray C, et al. Hesperidin displays relevant role in the nutrigenomic effect of orange juice on blood leukocytes in human volunteers: a randomized controlled cross-over study. PLOS One 2011;6:e26669.

21 Milenkovic D, Deval C, Gouranton E, et al. Modulation of miRNA expression by dietary polyphenols in apoE deficient mice: a new mechanism of the action of polyphenols. PLoS One 2012;7:e29837-167.

22 Milenkovic D, Jude B, Morand C. miRNA as molecular target of polyphenols underlying their biological effects. Free Radic Biol Med 2013;64:40-51.

23 Yahfoufi N, Alsadi N, Jambi M, et al. The immunomodulatory and anti-inflammatory role of polyphenols. Nutrients 2018;10. doi:10.3390/nu10111618. [Epub ahead of print: 02 Nov 2018].

24 Borkowski K, Yim SJ, Holt RR, et al. Walnuts change lipoprotein composition suppressing TNF $\alpha$-stimulated cytokine production by diabetic adipocyte. J Nutr Biochem 2019;68:51-8.

25 Gabbs M, Leng S, Devassy JG, et al. Advances in our understanding of oxylipins derived from dietary PUFAs. Adv Nutr 2015;6:513-40.

26 Burton-Freeman B. Postprandial metabolic events and fruit-derived phenolics: a review of the science. Br J Nutr 2010;104 Suppl 3:S1-14.

27 IFU. Determination of sodium, potassium, calcium and magnesium. In: Methods of analysis, international federation of fruit juice producers. vol. 58. Zug, Switzerland, 2005.

28 Aschoff JK, Kaufmann S, Kalkan O, et al. In vitro bioaccessibility of carotenoids, flavonoids, and vitamin $\mathrm{C}$ from differently processed oranges and orange juices [Citrus sinensis (L.) Osbeck]. J Agric Food Chem 2015;63:578-87.

29 IFU. Determination of Hesperidin and Naringin HPLC. In: Methods of analysis, International Federation of fruit juice producers. vol 58. Zug, Switzerland, 2005.

30 Widlansky ME, Hamburg NM, Anter E, et al. Acute EGCG supplementation reverses endothelial dysfunction in patients with coronary artery disease. J Am Coll Nutr 2007;26:95-102.

31 Heiss C, Kleinbongard P, Dejam A, et al. Acute consumption of flavanol-rich cocoa and the reversal of endothelial dysfunction in smokers. J Am Coll Cardiol 2005;46:1276-83.

32 Corretti MC, Anderson TJ, Benjamin EJ, et al. Guidelines for the ultrasound assessment of endothelial-dependent flowmediated vasodilation of the brachial artery: a report of the International brachial artery reactivity Task force. J Am Coll Cardiol 2002;39:257-65.

33 Mullen W, Archeveque M-A, Edwards CA, et al. Bioavailability and metabolism of orange juice flavanones in humans: impact of a full-fat yogurt. J Agric Food Chem 2008;56:11157-64.

34 Tessier F, Birlouez-Aragon I, Tjani C, et al. Validation of a micromethod for determining oxidized and reduced vitamin $\mathrm{C}$ in plasma by HPLC-fluorescence. Int $J$ Vitam Nutr Res 1996;66:166-70.

35 Mainka M, Dalle C, Pétéra M, et al. Harmonized procedures lead to comparable quantification of total oxylipins across laboratories. $J$ Lipid Res 2020;61:1424-36. 